\title{
PASSAGES TRANSLATED
}

Aristophanes

Acharnians

$$
\text { 524-53 I }
$$$$
\text { 532-539 }
$$

Clouds

$$
\begin{aligned}
& 2 \text { I I-2 I } 3 \\
& 858-859
\end{aligned}
$$

Knights

$$
283
$$$$
\text { I037-1038 }
$$

Peace

$$
\begin{aligned}
& 603-6 \text { I I } \\
& 6 \text { I5-6 I } 7
\end{aligned}
$$

Wasps

$$
\text { I I I 7-I I 2I }
$$

[Aristotle]

The Constitution

of the Athenians

$$
26.4-27 \cdot 3,28.1-3 \quad 2 \text { I-22 }
$$

Aristotle

Rhetoric

$$
\begin{array}{ll}
\text { I407a2-6 } & 29 \\
\text { I4 I Ia2-4 } & 29 \\
\text { I4 I Ia I5-I6 } & 29 \\
\text { I4 I9a2-5 } & 30
\end{array}
$$

Cratinus

Chirons

frag. $25^{8} \quad$ I05

frag. 259 I06 


\section{xii / Passages Translated}

Nemesis

frag. I 8

Thracian Women

frag. 73

Unnamed

comedy frag. 326
I 06

Io6

I05

Eupolis

Demes

frag. 102

28

frag. I 5

Hermippus

Moirai frag. $47 \quad \mathrm{IO} 4$

Herodotus

Histories

I. 4.3

I. 5 . I

$6.13 \mathrm{I}$

$I G \mathrm{I}^{3} 884$

Isocrates

Antidosis

$$
\text { 234-235 }
$$

On the Chariots

28
99

I 06

99

I IO

37

I 54

I 54
Lycurgus

Against Demades

frag. 2

I $54-55$

Lysias

Against Andocides

IO

I 29

Against Eratosthenes

4

I 28

Against Nicomachus

28

I 29-30

Plato

Apology

$$
\begin{array}{ll}
\text { I } 9 \text { c2-4 } & \text { I36 } \\
\text { I } 9 \text { c5-8 } & \text { I37 } \\
24 \text { b8-c I } & \text { I3 I } \\
26 \mathrm{~d}-\mathrm{e} & \text { I36 }
\end{array}
$$

Gorgias

$$
503 \mathrm{~b}-\mathrm{c} 6
$$

I38-39

5 5 е2-5 г6а3

I39

${ }^{\mathrm{I} 6 \mathrm{~b} 8}-\mathrm{d}_{4} \quad$ I39-40

$5 \mathrm{I} 7 \mathrm{~b} 2-\mathrm{c}_{3} \quad \mathrm{I} 4 \mathrm{O}-4 \mathrm{I}$

$5 \mathrm{I} 8 \mathrm{e}-5 \mathrm{Igd} 5 \quad \mathrm{I} 4 \mathrm{I}-4^{2}$

Phaedrus

269e

$270 \mathrm{a}$

I35 


\section{Passages Translated / xiii}

Protagoras

$$
329 \mathrm{a}
$$

Plutarch

Life of Pericles

8.7

8.8

8.9

I3

I 8.2

23.1

$33 \cdot 5$

$35 \cdot 2$

38.4

Protagoras

frag. 8o B 9

I I7

Sophocles

Oedipus the King

I-I 3

I 2 I

I $4-57$

$5^{8-69}$

Telecleides
Thucydides

History

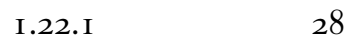

I.I27 48

I.I38.3 59

I.I39.4 27

I.I39-I $45 \quad 5^{\mathrm{I}-57}$

I.I4O.I $\quad 5$ I

I.I $43.5 \quad 30$

I.I44.I 50

$2.13 .2-14 \quad 62-63$

2.21.2-22.2 $\quad 65-67$

$2.34 .8-46 \quad 68-76$

2.4 I.I $\quad 77$

$2.43 \cdot 3 \quad 77$

2.53.2-4 80

2.56. 180

2.59-65.4 8I -87

$2.60 .5 \quad 88$

$2.65 \cdot 5-13 \quad 88-90$

Xenophon

Memorabilia

I. $2.40-46 \quad$ I $32-33$

$\begin{array}{ll}\text { frag. } 45 & \text { I0 } 4 \\ \text { frag. } 47 & \text { I0 } 4\end{array}$


This page intentionally left blank 